\title{
RETICULOCYTES AND THEIR HUMORAL REGULATION
}

\author{
BY \\ ERIK JACOBSEN \\ From the Biological Laboratories of Medicinalco Ltd., Copenhagen
}

(RECEIVED FOR PUBLICATION, JUNE, 1946)

The reticulocytes are generally accepted to be young red blood cells. Whenever red cell formation in the bone marrow is active the proportion of reticulocytes in the blood increases; hence the importance of the reticulocyte count in haematology. Nevertheless, there have been few studies dealing with purely physiological and chemical aspects. A study of the ripening of reticulocytes into mature non-reticulated red cells has, however, been carried out in our laboratory during the last five or six years. The present article reviews the results obtained.

\section{Experimental Procedure}

Rabbits were used as experimental animals. In order to obtain reasonable accuracy in counting their reticulocytes, the animals were brought into a chronic anaemic state by the daily withdrawal of from 30 to $50 \mathrm{ml}$. of blood. At the end of one week the haemoglobin percentage thus fell to 40 or 50 and the red cell count to 2 or 3 million per c.mm. of blood, with 20 or 30 per cent reticulocytes. This state could be maintained for a month or more before the animals died. The blood of such animals was used in the tests (Plum, 1942a).

Blood was centrifuged and the red cells washed in saline. They were then resuspended in saline, or in plasma or saline to which had been added the substance whose action on the reticulocytes was to be studied. The suspensions were kept in small test-tubes in a water bath at $40^{\circ} \mathrm{C}$. and gently and constantly moved. Samples for reticulocyte counts were taken at regular intervals.

Reticulocytes suspended in saline alone disappeared slowly, but the rate of disappearance could be accelerated considerably by adding commercial liver extract to the saline in which the cells were suspended (Fig. 1). The youngest and most unripe types of reticulocytes disappeared first, and as there was apparently no haemolysis this must have been due to their maturation into "adult" red cells. This process seemed to be accelerated by some substance found in liver extracts.

The ripening process followed the monomolecular equation well known in physical chemistry and biochemistry:

$$
\mathrm{k}=\frac{1}{\mathrm{t}} \log \frac{\mathrm{a}}{\mathrm{a}-\mathrm{x}}
$$

where $t$ is the time of incubation, $a$ the number of reticulocytes at the beginning of the experiment, and $x$ the number of reticulocytes that disappear during the time $t ; k$ is the "monomolecular constant." This formula only indicates that the same percentage of reticulocytes is ripened in the same interval of time regardless of the number of reticulocytes. The constant, however, gives a very convenient measure for the rate of ripening; the higher the constant, the faster the rate, and vice versa; $k$ increases with rising temperature.

Experiments with varying concentrations of liver extract have shown that the constant in the ripening

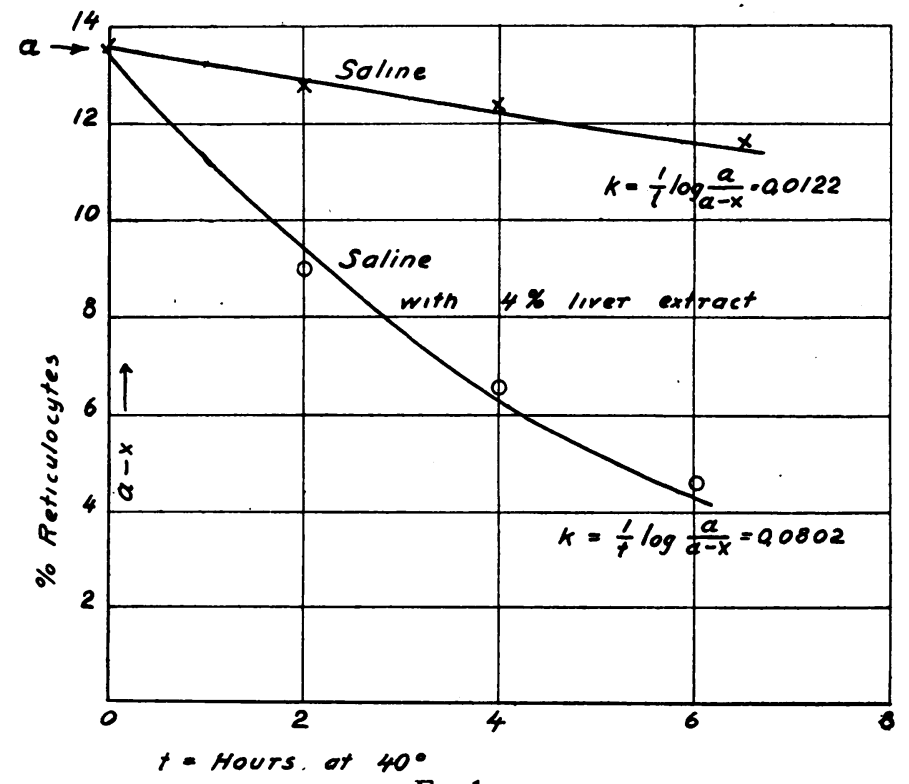

FIG 1. 


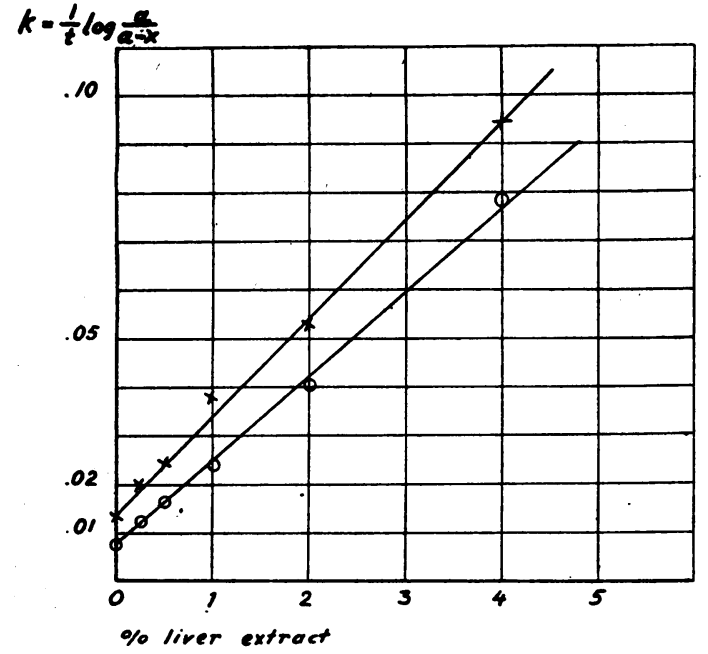

FIG. 2

experiments is directly proportional to the concentration of liver extract (Fig. 2).

\section{Chemical Nature of Reticulocyte-ripening Principle}

The reticulocyte-ripening principle in liver extract is thermolabile; it loses its activity after heating for five minutes on a boiling water bath. Thus it cannot be identical with the thermostable principle acting against pernicious anaemia. It has been shown, moreover, that the reticulocyte-ripening principle in liver consists of a thermolabile fraction which can be absorbed to floridine and a thermostable fraction that cannot be absorbed (Jacobsen and Plum, 1942). The latter fraction has little effect by itself, but activates the effect of the thermolabile component. The thermostable fraction can be extracted from liver extract with butanol and isolated from the butanol extract. It has been

TABLE I

\section{Reticulocyte ripening principle}

thermolabile fraction
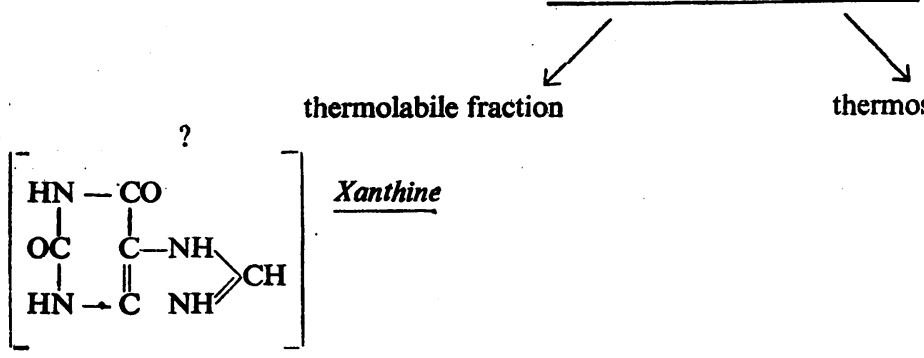

Xanthine

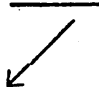

$$
\text { n }
$$

thermostable fraction<smiles>N=C1C=C(NC(=O)Cl)C(=O)N1</smiles>

\section{Leucopterin}

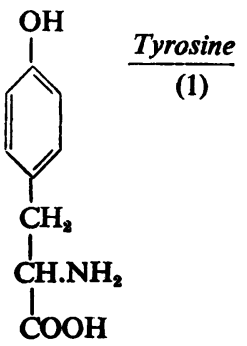<smiles>N=c1[nH]c(=O)c2nc(CNc3ccc(C(=O)NC(CCC(=O)O)C(=O)O)cc3)cnc2[nH]1</smiles><smiles>C#CCCCC(=O)O</smiles>

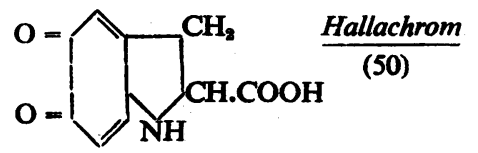


found to be identical with 1-tyrosine. Synthetically prepared tyrosine shows exactly the same effect in combination with the thermolabile fraction.

The effect of tyrosine seems to be specific. None of the thirteen most common amino acids, including phenylalanine, has any activating effect on reticulocyte ripening, but compounds similar to tyrosine, such as adrenaline and tyramine, have some effect (Jacobsen and Plum, 1942). Later investigations have shown that certain oxygenated products of tyrosine, dioxyphenylalanine and hallachrom, have a much stronger activating effect, that of hallachrom being 50 to 100 times stronger than that of tyrosine (Gad and others, 1944). As there are enzymes present in red cells capable of converting tyrosine into hallachrom it is possible that the activating effect is not due to tyrosine, but to hallachrom derived from it.

The thermolabile reticulocyte-ripening fraction has not been isolated from liver. Systematic investigations on various organs have shown, however, that stomach tissue (Plum, 1944a), duodenum, and the upper part of the small intestine contain considerable amounts of the thermolabile fraction (Bohn, 1946). Extracts from these tissues have little ripening activity by themselves, but marked ripening effects, even greater than that of liver extracts, have been found after the addition of tyrosine, dioxyphenylalanine, or hallachrom. The ripening fraction in gastric tissue is extremely labile; however, a rather powerful preparation

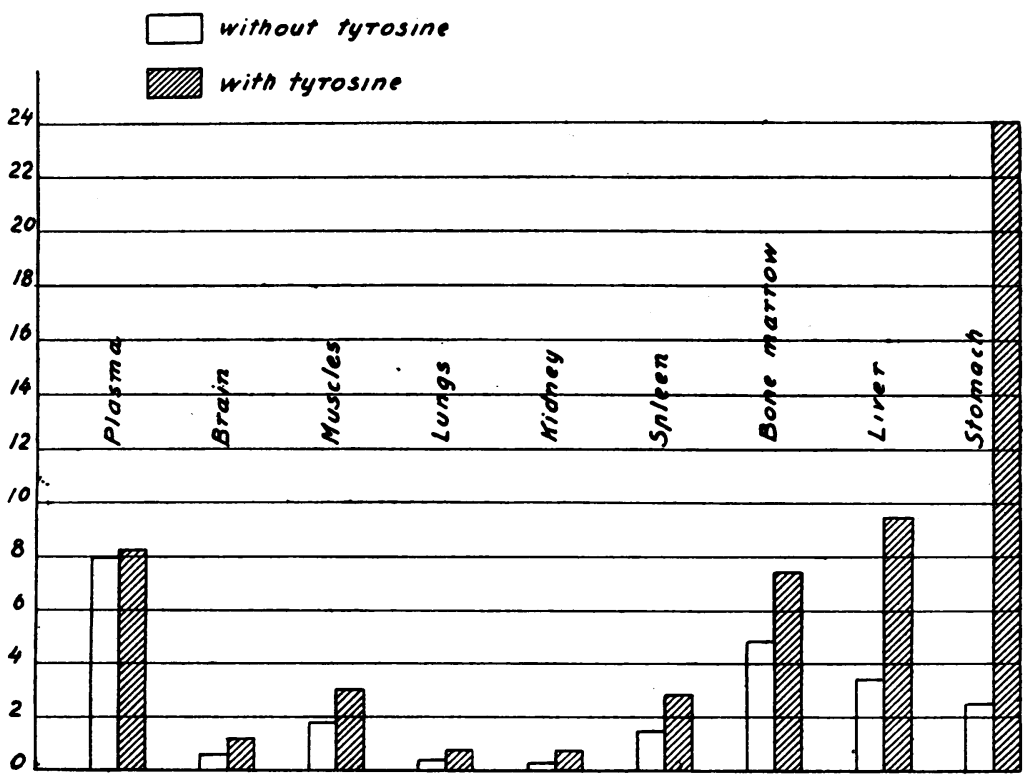

FIG. 3 containing 75 to 80 per cent xanthine has been isolated from it (Jacobsen, 1944). Synthetically prepared xanthine in combination with tyrosine has a marked effect, but it does not seem likely that the effect of the gastric extract can be explained by its content of xanthine. Probably a tautomeric isomer is responsible. Some chemical analogues of xanthine have been tested. Guanine and caffeine amongst others are ineffective. Recently both leucopterin and folic acid have been shown to be more potent than xanthine, although with qualitative differences. Xanthine is only effective in the presence of tyrosine and is thermolabile. Leucopterin and folic acid have, however, an effect even without tyrosine, but are activated 100 per cent or more after its addition. The potency of neither of these compounds is affected by boiling.

The chemical problem has not been solved. Several experiments indicate that the thermolabile fraction consists of more than one substance. The fact that substances which have recently been shown to have an effect on haemopoiesis in vivo may accelerate ripening of reticulocytes in vitro is, nevertheless, of great interest.

\section{CONTENT OF RETICULOCYTE-RIPENING FACTORS IN Organs and Plasma}

As has already been stated, reticulocyte-ripening principles may be found in extracts from several organs of the body. Calculated per gramme of tissue, the greatest concentration is found in plasma, and slightly less in bone marrow, liver, stomach, and spleen (Plum, 1944a). Extracts of all these organs, but not plasma, may be activated by the addition of tyrosine (Fig. 3). The stomach and the upper intestinal tract contain the highest concentration of the thermolabile fraction. Large amounts are found in the duodenum and in the upper part of jejunum, apparently following the distribution of Brunner's glands. The results of chemical investigations on stomach extracts have already been mentioned. In Fig. 3 is shown the distribution in swine ; similar relative distributions are found in the organs of oxen, rats, rabbits, and guinea-pigs. 
Unlike liver extracts the reticulocyte-ripening principle in plasma cannot be activated by tyrosine, and plasma is not able to activate the thermolabile fraction further as it ought to if it contained tyrosine or tyrosine-likesubstances. However, when plasma is treated with acetone, the ripening principle is transformed into a state in which it can be activated by tyrosine. This shows that there is no great difference between the ripening principles in plasma and liver; the linkage between thermolabile and thermostable fractions in plasma must be a strong one.

\section{Role of THE Reticulo-Endothelial System}

Experiments with rabbits have shown that the action of the thermostable component is influenced by the activity of the reticulo-endothelial system. The content of ripening substances in plasma has been determined with and without the addition of tyrosine. After a control period the reticulo-endothelial system was blocked by intravenous injection of $10 \mathrm{ml}$. 1 per cent trypan blue combined with splenectomy. The activity of the reticulo-endothelial system, as tested for by the disappearance of congo red, rapidly drops to zero and recovers slowly, becbming normal again by the sixteenth to eighteenth day. Fig. 4 demonstrates that the amount of ripening principle in plasma closely follows the activity of the reticuloendothelial system; after addition of tyrosine, however, there is no difference from the normal. Blocking has thus no influence upon the thermolabile component.

These findings make it seem probable that tyrosine (or a derivative of, or an oxygenated product of, tyrosine) and the thermolabile substance are linked in the reticuloendothelial system and together form

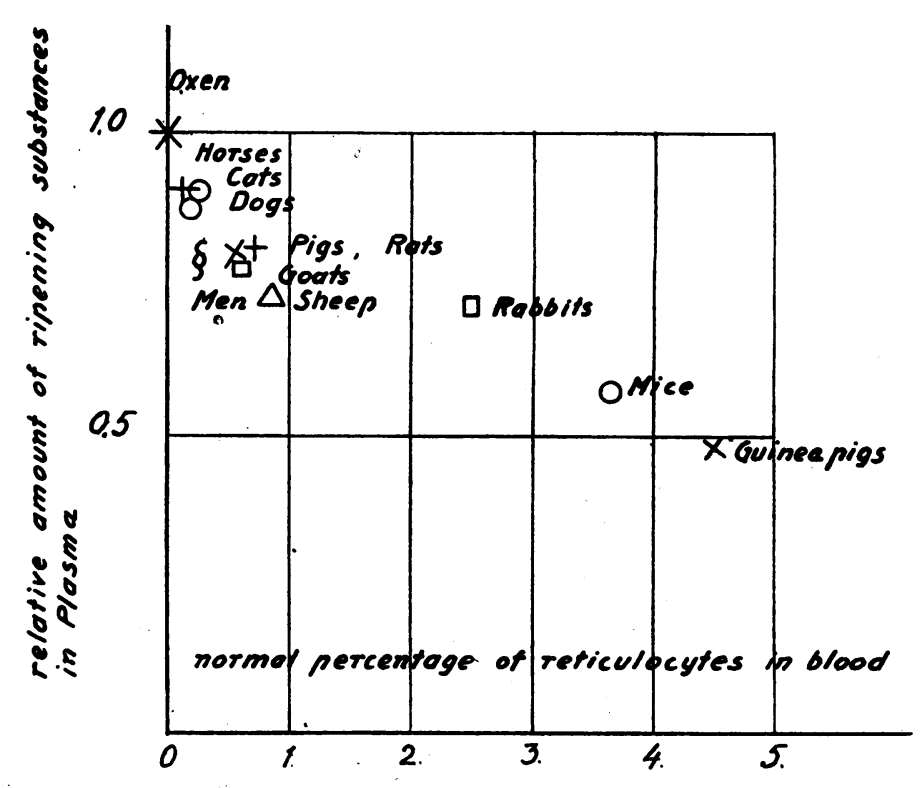

FIG. 5

the reticulocyte-ripening principle found in plasma (Jacobsen and Plum, 1943a).

Content of Reticulocyte-ripening Principles in the Plasma of Various Animals and Man

As the content of the ripening factors in organs varies with that in plasma, most of the investigations 
have been carried out on the plasma of various animals. The amount of reticulocyte-ripening factor in plasma varies in different animals (Plum 1942b, 1943). Reptiles and birds have practically none. In mammals there is considerable variation (Fig. 5). The plasma of animals whose blood normally contains few or no reticulocytes (for example, oxen) seems to contain the highest content of ripening substances, whilst that of species whose blood normally has a relatively high percentage of reticulocytes (e.g., guinea-pigs) has the lowest content, in the case of guinea-pigs half that of oxen.

The content of ripening substances in the plasma of normal adults also seems to vary inversely with the reticulocyte count. In certain physiological and pathological states, however, this relationship may not hold (Table II).

TABLE II

\begin{tabular}{|c|c|c|}
\hline & Reticulocytes & $\begin{array}{l}\text { Ripening } \\
\text { substances }\end{array}$ \\
\hline Early life $\quad \ldots$ & +++ & +++ \\
\hline 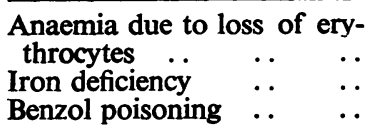 & $\begin{array}{l}++ \\
+ \\
+\end{array}$ & $\begin{array}{l}+ \\
+ \\
+\end{array}$ \\
\hline $\begin{array}{c}\text { Gastrectomy or duodenec- } \\
\text { tomy } \ldots \\
\text { Pernicious anaemia }\end{array}$ & $\stackrel{(+)}{?}$ & 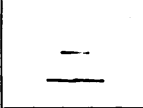 \\
\hline $\begin{array}{lll}\text { Hypothyroidism } & . & \ldots \\
\text { Hyperthyroidism } & \cdots & \ldots \\
\text { Hypophysectomy } & \cdots & \ldots\end{array}$ & 0 & $\bar{z}$ \\
\hline Menstruation & $(+)$ & $(+)$ \\
\hline $\begin{array}{ccc}\text { Jaundice, obstructive } & \text { and } \\
\text { haemolytic } . . & \ldots & \ldots\end{array}$ & $(+)$ & $(+)$ \\
\hline
\end{tabular}

In foetal life and in infancy until the blood picture becomes normal, the increased numbers of reticulocytes are associated with a considerably increased amount of ripening principles. In newborn rats or rabbits with 80 or 100 per cent of their red cells in the form of reticulocytes, about double the normal concentration of ripening substances may be found. Both reticulocyte percentage and content of ripening substances decrease gradually and reach normal values at the same age (Plum, 1943). A probable explanation of the occurrence of high reticulocyte percentages and high concentrations of ripening substances, for which there is experimental support, is that the immature blood corpuscles in the early ages react more slowly to humoral stimulation (Jacobsen and Plum, 1943b).
The reticulocytosis associated with increased blood formation, as seen in chronic post-haemorrhagic anaemia in rabbits, is always combined with an increase in ripening substances (Plum, 1943). In this case the response of the reticulocytes to a concentration of ripening substances does not change, but the quantity of ripening substances in the plasma increases. This increase may cause the reticulocytes in the peripheral blood stream of anaemic rabbits to ripen about 20 or 30 per cent more rapidly than in normal animals. The same phenomenon is seen when anaemia is caused by some haemolytic poison such as phenylhydrazine (Plum, 1944b).

Similarly, in iron-deficiency anaemia of dietary origin reticulocytosis is accompanied by an increased concentration of reticulocyte-ripening substances (Plum, 1944b). On treatment with iron, both anaemia and reticulocytosis disappear and the content of reticulocyte-ripening substances in the plasma returns to normal. Anaemia and leucopenia result from the effects of benzol poisoning on the bone marrow. The reticulocyte count may fall or rise slightly, but the content of reticulacyte-ripening substances in the plasma is always increased. During regeneration with increases in leucocytes and reticulocytes the content of ripening substances returns to normal. These experiments suggest that the rise in reticulocyte-ripening substances may be interpreted as one of the measures by which the body attempts to compensate for anaemia.

Occasionally an increased number of reticulocytes is associated with reduced amounts of reticulocyteripening principles in the plasma, as in rats after gastrectomy (unpublished observations) and in swine in which the duodenum had been either isolated or excised causing a 20 per cent decrease in activity (Bohn and others, 1945).

In untreated pernicious anaemia a decrease up to 40 per cent has been observed. The addition of tyrosine has in all cases raised the concentration of ripening substances up to the normal level. This decrease is thus not due to a decreased concentration of the thermolabile component, as might have been expected from the known high concentration of the thermolabile fraction in the gastric tissue of the normal subject and the characteristic gastric atrophy in pernicious anaemia.

Both in spontaneous hypo- and hyperthyroidism in man and in the experimental animal there is a decreased content of ripening substances in the plasma, which may be corrected by the addition of tyrosine. The decrease after hypophysectomy may result from decrease in the function of the thyroid gland. 
During menstruation a slight increase has been observed starting on the first day.

Obstructive jaundice in patients and in animals may be associated with a slight increase in ripening substances.

\section{Conclusion}

Assay of the concentration of reticulocyte-ripening substances in the plasma is of more scientific than practical interest. It has no diagnostic value. The plasma of from one hundred to one hundred and fifty patients with various diseases and that of about fifty normal persons have been tested. A definite decrease in the content of ripening substances in the plasma has been found in untreated pernicious anaemia, in Graves's disease, in some patients with gastric diseases, and in cachectic patients suffering from cancer (Plum, R., 1947). In all cases this decrease has been due to lack of the thermostable fraction and could be rectified by the addition of tyrosine. In some patients with jaundice the content of the ripening substances has been increased. In all other diseases normal levels have been found.

Even a slight reticulocytosis has been generally considered a sign of increased erythropoiesis. The work now described indicates that this may not always be the case. The reticulocyte percentage in peripheral blood depends upon the output of reticulocytes from the bone marrow, controlled by factors still unknown, and the rate of ripening in the blood stream, the latter regulated by the ripening principles. Thus with a constant production the numbers of reticulocytes in the circulating blood can increase if the concentration of ripening substances in plasma decreases, and vice versa. Slight reticulocytosis cannot, therefore, be regarded as a sign of increased or altered blood production unless it is certain that the concentration of ripening substances has not altered.

It is difficult to make any definite statement with regard to the physiological role of the ripening substances, particularly as it is doubtful what reticulocytes really are. It is thought that under normal conditions not all the red cells released from the bone marrow are in the form of reticulocytes. As the capacity of the reticulocytes to transport oxygen is as good as that of adult cells, it is doubtful whether their maturation into adult corpuscles is of any great physiological significance. The as yet unpublished observations of my collaborator, Dr. C. M. Plum, who has done a major part of the work described in this paper, seem to show that a similar mechanism may operate in the formation of red cells from normoblasts. If this is true, the ripening of reticulocytes can be regarded as a special case or model of the course of erythropoiesis as a whole. The demonstration of a humoral regulation of blood-cell formation may be expected to throw new light on many of the hitherto unsolved problems in erythropoiesis.

\section{Summary}

A principle capable of accelerating the ripening of reticulocytes in vitro can be demonstrated in plasma and in various tissues of the body.

This principle consists of at least two fractions: a thermostable one, identified as tyrosine or tyrosine derivatives, and a thermolabile one. Xanthine, leucopterin, and folic acid are able to act as the thermolabile factor. The greatest concentration of the thermolabile fraction is found in the stomach and duodenum. The thermolabile and thermostable fractions linked together by the activity of the reticulo-endothelial system form the principle found in plasma.

An increased amount of ripening principle may be found in the plasma in some cases of increased erythropoiesis; in decreased erythropoiesis a lower content than normal may be encountered. It seems as if the number of reticulocytes in the blood varies inversely with the amount of ripening principle, at least under normal conditions.

The significance of these findings is discussed.

\section{REFERENCES}

Bohn, C. L. S. (1946). " “Undersögelser over de Brunnerske Duodenelkirtlers Betydning for Erythropoesen hos Svinet." Copenhagen. Bohn, C. L. S., Landboe-Christensen, E., and Plum, C. M. (1945). Acta med. scand., 120, 32.

Gad, I., Jacobsen, E., and Plum, C. M. (1944). Acta physiol. scand., T, 244.

Jacobsen, E. (1944). Acta physiol. scand., 7, 342.

Jacobsen, E., and Plum, C. M. (1942). Acta physiol. scand., 4, 272, 278. Jacobsen, E., and Plum, C. M. (1943a). Acta physiol. scand., 5, 1.

Jacobsen, E., and Plum, C. M. (1943b). Klin. Wschr., 22, 491.

Plum, C. M. (1942a). Acta physiol. scand., 4, 260.

Plum, C. M. (1942b). Acta med. scand., 112, 151.

Plum, C. M. (1942b). Acta med. scand., 112, 151. 175.

Plum, C. M. (1944a). "Reticulocytmodmingen in Vitro." Copenhagen.

Plum, C. M. (1944b). Acta med. scand., 117, 29.

Plum, Ruth (1947). "Reticulocytmodnings Index." Thesis. Copenhagen. (In Danish.) 